investigate the left ventricular diastolic function and the factors related in SLE patients compared with healthy controls.

Methods Thirty consecutive female SLE patients without evidence of cardiac disease were underwent standard transthoracic echocardiography, and were compared with 30 agematched healthy female controls. Patient characteristics, organ damage and laboratory data were retrieved by medical chart review.

Results In SLE patients, indexes of LV diastolic function differed from control group, with reduced early diastolic filling velocity (E), as well as prolongation of the time taken from the maximum $\mathrm{E}$ point to baseline, reduced ratio of early to late diastolic flow velocity $(\mathrm{E} / \mathrm{A})$, prolonged ratio of $\mathrm{E}$ to early diastolic mitral annular velocity (E') (E/E'). However, the differences did not show statistical significance. Anti-Ro antibody positivity was observed in $43 \%$ of SLE patients, and it was correlated with higher E/A ratio significantly $(1.3 \pm 0.4$ vs 1.0 $\pm 0.2, \mathrm{p}=0.03$ ). In addition, the SLE patients with hematologic or renal involvement showed more enlarged size of left atrium significantly compared to the patients without any involvement $(36 \pm 4.3$ vs $31 \pm 9.2, p=0.01)$.

Conclusions Although not statistically significant, there was a trend which suggested that patients with SLE have subclinical impaired diastolic function compared with the healthy control. Presence of anti-Ro antibody and systemic organ involvement was related with the diastolic dysfunction markers.

\section{EDA+ FIBRONECTIN IN TUBULO-INTERSTITIAL INJURY IN LUPUS NEPHRITIS}

HK Choy, S Yung, N Li, KF Cheung, TM Chan. The University of Hong Kong, Medicine, Hong Kong, Hong Kong S.A.R

\subsection{6/lupus-2017-000215.208}

Background and aims Anti-dsDNA antibody plays a critical role in the pathogenesis of lupus nephritis and contributes to inflammatory and fibrotic processes in the kidney. $\mathrm{EDA}^{+}$ spliced variant of fibronectin $\left(\mathrm{EDA}^{+} \mathrm{FN}\right)$, normally only weakly expressed, is markedly increased in pathological conditions. We investigated the effect of human polyclonal antidsDNA antibodies on the expression of $\mathrm{EDA}^{+} \mathrm{FN}$ in proximal tubular epithelial cells (PTEC) and the functional consequence. Methods $\mathrm{EDA}^{+} \mathrm{FN}$ expression in human renal biopsies of Class III/IV $\pm \mathrm{V}$ lupus nephritis was assessed by cytochemistry. Cultured PTEC were incubated with control IgG or IgG antidsDNA antibodies isolated from lupus nephritis patients for 24 hour and the expression of $\mathrm{EDA}^{+} \mathrm{FN}$ was investigated. Recombinant human EDA peptide was used to investigate the functional role of $\mathrm{EDA}^{+} \mathrm{FN}$ in PTEC.

Results The results showed that $\mathrm{EDA}^{+} \mathrm{FN}$ was absent from normal kidney tissue but was markedly increased in the tubulo-interstitium in lupus nephritis patients. Cultured PTEC constitutively expressed native $\mathrm{FN}$ but not $\mathrm{EDA}^{+}$FN. AntidsDNA antibodies, compared with serum-free-medium and control IgG, increased $\mathrm{EDA}^{+} \mathrm{FN}$ expression by 5.8- and 5.6fold respectively $(\mathrm{p}<0.05$ for both), and the induction was mediated through PI3K and mTOR activation. Exogenous IL$1 \beta$ and TGF- $\beta 1$, but not IL- 6 , IL-8 or MCP-1, induced EDA ${ }^{+}$ FN by 1.8 - and 2.3-fold respectively. Recombinant EDA peptide increased native $\mathrm{FN}$, collagen I, laminin and SNAIL expression, but decreased E-cadherin expression, in PTEC.
Conclusions Our data demonstrated a role of $\mathrm{EDA}^{+} \mathrm{FN}$ in the pathogenesis of tubulo-interstitial disease in lupus nephritis.

\section{9 \\ JOINT DEPOSITED IGG INDUCES ARTHRITIS BUT INHIBITS OSTEOCLASTOGENESIS IN SLE THROUGH SYK}

GM Deng, W Qiao, H Ding, H Liu, X Guo. Nanjing Medical University, Key Laboratory of Antibody Techniques of Ministry of Health, Nanjing, China

\subsection{6/lupus-2017-000215.209}

Background and aims Although arthritis is frequent in patients with systemic lupus erythematosus (SLE), its pathogenesis remains unclear. The aim in our study is to investigate the pathognesis of arthritis in SLE.

Methods We analysed the feature of SLE patients with arthritis and lupus-prone mice with arthritis, investigated the role of joint deposited $\operatorname{IgG}$ in the development of lupus arthritis.

Results Arthritis lacking bone erosion is common symptom in most of SLE patients and spontaneously develops in lupus prone mice. Large amount of $\operatorname{IgG}$ deposited in joint of lupus prone mice. Similar arthritis to lupus prone mice was induced by intraarticular injection of lupus $\operatorname{IgG}$ and was dependent on the dose of lupus IgG. Joint deposited IgG, monocytes/macrophages and TNFa were required in the development of lupus arthritis. Joint deposited lupus IgG inhibited RANKL-induced osteoclastogenesis in dose and time dependent manner. Lacking ITAM containing FcyRIII reduced inhibitory effect of lupus IgG on osteoclastogenesis. Lupus IgG quickly stimulated Syk activation than RANKL through lipid rafts. Lupus IgGinduced Syk activation is related to dsDNA Ab. Blocking of Syk significantly inhibited arthritis induced by lupus IgG and arthritis in lupus prone mice, suppressed Syk activation induced by lupus IgG and osteoclastogenesis induced by RANKL.

Conclusions The joint deposited IgG exerts an important role in the development of lupus arthritis lacking of bone destruction, Syk plays a crucial role in lupus IgG-induced arthritis and inhibited osteoclastogenesis. This finding will promote development of effective therapeutic strategy to arthritis in SLE patients.

\section{OESTROGEN PROMOTES SLE SERUM IGG-INDUCED SKIN INFLAMMATION VIA THE OESTROGEN MEMBRANE RECEPTOR GPER 1}

Z Cai, GM Deng. Nanjing Medical University, Key Laboratory of Antibody Techniques of Ministry of Health, Nanjing, China

10.1136/lupus-2017-000215.210

Background and aims Skin injury is the second most common clinical manifestation in patients with systemic lupus erythematosus (SLE). Oestrogen may affect the onset and development of SLE. This study was undertaken to elucidate the role of oestrogen in the development of SLE skin injury.

Methods We investigated the role of oestrogen and its membrane receptor GPER1 in SLE-related skin injury in mice treated with SLE serum in vivo, and monocytes from mouse spleen in vitro.

Results We found that skin injury induced by SLE serum was more severe in female mice and required monocytes. E2 